\title{
Fuzzy Finite Difference Scheme to Simulate Radon Transport from Subsurface Soil to Buildings
}

\author{
P. S. Sharma and D. Datta* \\ Health Physics Division, Bhabha Atomic Research Centre, Mumbai - 400 085, \\ India \\ *Corresponding author (e-mail: ddatta@barc.gov.in)
}

\begin{abstract}
In this paper, a methodology for solving the advection diffusion equation describing the radon transport from subsurface soil into buildings in presence of the imprecision in the measurements of model parameters such as radon diffusion coefficient and flow velocity of radon in air has been explored. Imprecision of the model parameters is addressed as a fuzzy variable and the membership function of each such fuzzy variable is expressed in the form of a triangular fuzzy number. Explicit finite difference numerical method along with fuzzy parameters of the representative model is applied to obtain the solution of the governing advection diffusion equation and due to this the present numerical approach is named as fuzzy finite difference. Uncertainty quantification of the radon concentration as solution of the governing fuzzy partial differential equation is carried out and by using this uncertainty modeling, advantage of the fuzzy finite difference approach for obtaining the numerical solution of a fuzzy partial differential equation is shown. Results of computed radon concentration with uncertainty can be possible to use for assessing further the uncertainty in health hazards.
\end{abstract}

Keywords: Fuzzy number; uncertainty; advection diffusion; imprecision; radon; finite difference

\section{Introduction}

Radon with its short half-life progeny $\left({ }^{222} \mathrm{Rn},{ }^{220} \mathrm{Rn}\right.$ and $\left.{ }^{219} \mathrm{Rn}\right)$ is the main contributor of $50 \%$ of the natural radiation dose received by people. Therefore, it is mandatory to pay more attention on the issue of health risks from them. Basically, radon in the ambient atmosphere is mainly released from the radioactive elements uranium and radium in the soil and rocks underground. Therefore, controlling of radon is significant for delineation of the potential high radon zone in presence of the local geological background. Radon progeny belong to the natural decay chains of ${ }^{238} \mathrm{U},{ }^{232} \mathrm{Th}$ and ${ }^{235} \mathrm{U}$ respectively. The nuclide ${ }^{222} \mathrm{Rn}$ among others is the longest-lived with a half life of 3.825 days (decay constant, $\lambda_{\mathrm{Rn}}=2.1 \times 10^{-6} \mathrm{~s}^{-1}$ ). A large number of geochemical data such as the uranium content and the distribution of soil texture are available which can be used along with radon migration theory to predict the regional radon distribution. In a built environment, ${ }^{222} \mathrm{Rn}$ emanates from soil. It is also a fact that building materials and some utility supply (water and gas) are the sources of ${ }^{222} \mathrm{Rn}$. It was reported that a worldwide average of $60.4 \%$ of the total indoor ${ }^{222} \mathrm{Rn}$ originates from the surroundings and underground of the buildings [1]. Entry route of ${ }^{222} \mathrm{Rn}$ is mainly by diffusion and advection through cracks occurred in the building materials. Therefore, consequence of this physics results a very high indoor ${ }^{222} \mathrm{Rn}$ concentrations (up to $10^{4} \mathrm{~Bq} / \mathrm{m}^{3}$ ) [2]. On the basis of a steady-state radon transport model in porous medium [3] admixture with the local geological and pedagogical parameters, various methodologies for predicting a regional radon concentration are elsewhere found in literatures [4-6]. 
Physics of the advection and diffusion characteristics of the soil and building materials generates the knowledge base of the total indoor ${ }^{222} \mathrm{Rn}$ concentration and associated health hazards or risks. However, measured parameters such as diffusion coefficient of the soil and flow velocity of radon in air are imprecise either due to lack of measurements (few samples) or low precision measuring instruments used for experiment. Imprecise parameters are generally represented as fuzzy and therefore, the imprecision or fuzziness of the parameters (diffusion coefficient and flow velocity) results an uncertainty (epistemic) in the radon concentration and corresponding health hazard or risks. A large number of experimental researches have been reported for the transport of radon $\left({ }^{222} \mathrm{Rn}\right)$ through soil [7-11]. Transport of ${ }^{222} \mathrm{Rn}$ through a variety of medium has been modeled by transport equation [7-11]. Transport equation of ${ }^{222} \mathrm{Rn}$ is represented as advectiondiffusion equation. Numerical solution of this transport equation with deterministic value of diffusion coefficients and flow velocity has been solved using finite element method (FEM)[12] or finite difference method (FDM) [13]. It is not possible to get any clear picture about the superiority of FEM over FDM; however, FEM may be better suited for complex three dimensional geometries. FDM is generally applied for solving one dimension problem.

In this paper, we have proposed the numerical solution of one dimensional transport equation of ${ }^{222} \mathrm{Rn}$ with fuzziness of the diffusion coefficient and flow velocity using traditional forward time central difference (FTCD) finite difference scheme. A Computer code 'FUZYRAD (version 1.0)' has been developed for computing the radon concentration in presence of fuzziness of the parameters of the governing partial differential equation. The code FUZYRAD is written in Visual FORTRAN. In fact, fuzziness of the representative parameters forms the fuzzy partial differential equation describing the transport of radon $\left({ }^{222} \mathrm{Rn}\right)$ through subsurface soil into buildings. The remaining part of the paper has been organized into the following way. Section 2 describes the physics of the radon transport from subsurface soil to buildings which is required to formulate the governing equation of radon transport. Section 3 presents the fuzzy finite difference method to obtain the numerical solution of the governing fuzzy partial differential equation that describes the transport of radon. Section 4 presents the algorithm of uncertainty modeling of ${ }^{222} \mathrm{Rn}$ transport in presence of fuzziness of the diffusion coefficient and flow velocity of radon. Section 5 presents the corresponding results and discussions and finally section 6 concludes by highlighting the utility of fuzziness in uncertainty analysis.

\section{Physics of Radon Transport}

Literature study reveals that the source region of indoor radon is limited to some meters below the building. The physics of the radon transport from subsurface soil into buildings can be found in detail elsewhere in [14]. With a view to this literature study and to have the basis of the governing equation describing the radon transport, we present a short note about the mechanism of radon transport. Radon $(\mathrm{Rn})$ is a radioactive gas with three naturally occurring isotopes that flow in air pore space of the subsurface and is subject to radioactive decay. The half life for ${ }^{222} \mathrm{Rn}$ is 3.8 days, for ${ }^{220} \mathrm{Rn} 55 \mathrm{~s}$ and for ${ }^{219} \mathrm{Rn} 4 \mathrm{~s}$, respectively. Due to their very fast decay, ${ }^{220} \mathrm{Rn}$ and ${ }^{219} \mathrm{Rn}$ can only exceptionally cause radiation exposure problems. Therefore, throughout the manuscript the term "radon" will refer to ${ }^{222} \mathrm{Rn}$.

Radon is generated in the subsurface. The generation rate in a dry (not water saturated) underground is given by

$$
G=f \rho_{s} A_{R a} \lambda(1-\phi) / \phi
$$

where, $f$ signifies the emanation coefficient, $\rho$ represents the density of soil $\left(\mathrm{kg} / \mathrm{m}^{3}\right), A_{R a}$ is ${ }^{226} \mathrm{Ra}$ content in soil $(\mathrm{Bq} / \mathrm{kg}), \lambda=2.1 \times 10^{-6} \mathrm{~s}^{-1}$ is the decay constant of ${ }^{222} \mathrm{Rn}$ and $\phi$ 
signifies the porosity of soil. The spatial difference of radon concentration that exists between the surface $(\mathrm{x}=0)$ and the underground induces a diffusive radon flow towards the surface. An advective radon flow can also exist if a spatial air pressure difference exists in the subsurface. In the reference [14], it has been discussed possible origins for an air flow field in the underground. According to the reference [14], it can be concluded that the advective flux is mostly due to temperature differences between the free surface and the building, due to wind or due to ventilation and heating inside the house.

Considering these transport mechanisms, the rate of change of radon concentration in soil pore air can be modeled by the general transport equation

$$
\frac{\partial C}{\partial t}=\nabla \cdot(D \nabla C)-v \nabla C-\lambda C+G
$$

where, $\mathrm{C}$ represents the radon concentration, $\mathrm{D}$ signifies the radon diffusion coefficient, $v$ represents the flow velocity of radon in air, $\lambda$ is the radon decay constant and $\mathrm{G}$ represents the radon generation rate (source term) as given in Eq. (1). The terms in the right hand side of Eq. (2) addresses the physical phenomenon of diffusion, advection, decay and source term respectively. The mean air velocity can be described by a scaling with the porosity to

$$
v=\frac{1}{\phi}\left(\frac{k}{\mu} \nabla P\right)
$$

where, $\mathrm{k}$ is the permeability $\left(\mathrm{m}^{2}\right), \mu$ is the dynamic viscosity of air $(\mathrm{Pa} \mathrm{s})$ and $\mathrm{P}$ is the pressure $(\mathrm{Pa})$. For the pressure field the Laplace equation can be assumed:

$$
\nabla\left(\frac{k}{\mu} \nabla P\right)=0
$$

\section{Fuzzy Finite Difference Method}

In this work, we have numerically solved one dimension form of Eq. (2) with the fuzzy parameters such as diffusion coefficient, $\mathrm{D}$, the radon-bearing advective air velocity, $\mathrm{v}$ in soil, radon emanation coefficient, $\mathrm{f}$, density of the soil, $\rho$, activity content of radon in soil, $A_{R a}$, and porosity of the soil. Fuzziness of all these fuzzy parameters are expressed in the form of a triangular fuzzy number to maintain the measurement uncertainty expressed as mean \pm standard deviation. Considering the fuzziness of the parameters of the governing equation (Eq.(2)), we can rewrite Eq.(2) in its one dimension form as

$$
\frac{\partial \tilde{C}}{\partial t}=\tilde{D} \cdot \frac{\partial^{2} \tilde{C}}{\partial x^{2}}-\tilde{v} \frac{\partial \tilde{C}}{\partial x}-\lambda \tilde{C}+\tilde{G},
$$

where, the radon concentration is signifying as a fuzzy number due to obvious reason. The tilde, ' $\sim$ ' sign notifies the variable as fuzzy variable and distinguishes them from their classical (crisp) counterpart. In order to have the numerical solution of this fuzzy partial differential equation (Eq. (5)), we have to first remove its fuzziness and that can be done by considering the alpha cut value of the associated fuzzy numbers, $\widetilde{D}, \widetilde{v}$ and $\widetilde{G}$. Before to proceed further, it is required to have a short description about a fuzzy set and its alpha cut representation because alpha cut representation of a fuzzy set has been used in the numerical analysis. The details about a fuzzy set can be found elsewhere in [15], however, a short description pertaining to the definition of a fuzzy set, its alpha cut representation and some algebraic properties as required for finite differencing scheme is presented in the subsequent paragraphs. 


\subsection{Alpha cut and algebraic properties of fuzzy set}

Definition 3.1.1 Let $\mathrm{X}$ is a collection of objects denoted generically by $\mathrm{x}$. A fuzzy set $\tilde{A}$ in $\mathrm{X}$ is defined as a set of ordered pairs:

$$
\widetilde{A}=\left\{\left(x, \mu_{\tilde{A}}(x)\right) \mid x \in X\right\}
$$

where, $\mu_{\tilde{A}}(x)$ is called the membership functions or grade of membership of $\mathrm{x}$ in $\tilde{A}$. The range of the membership function is a subset of the nonnegative real numbers whose supremum is finite. As an example, let the fuzzy set, $\tilde{A}$ is represented as $\{0.1 / 2+0.4 / 5+$ $0.5 / 8+1.0 / 10+0.7 / 12.0+0.8 / 20\}$.

Definition 3.1.2 The $\alpha$-cut of a fuzzy set $\tilde{A}$ is defined as the set of elements that belong to the fuzzy set $\tilde{A}$ at least to the degree $\alpha$. For example, $A_{\alpha=0.5}=\{8,10,12,20\}$. Therefore, mathematical representation of $\alpha$-cut of a fuzzy set $\tilde{A}$ can be written as $A_{\alpha}=\left\{x \in X \mid \mu_{\tilde{A}}(x) \geq \alpha\right\}=\left[A_{L}, A_{U}\right]$. So, $\alpha$-cut of a fuzzy set is represented as a closed interval.

Definition 3.1.3 The triangular fuzzy number $\widetilde{B}$ is defined by three numbers $\beta<\gamma<\delta$ as follows:

$$
\widetilde{B}=(\beta, \gamma, \delta)
$$

The membership function representation of this triangular fuzzy number, $\widetilde{B}$ (Fig. 1) is given by

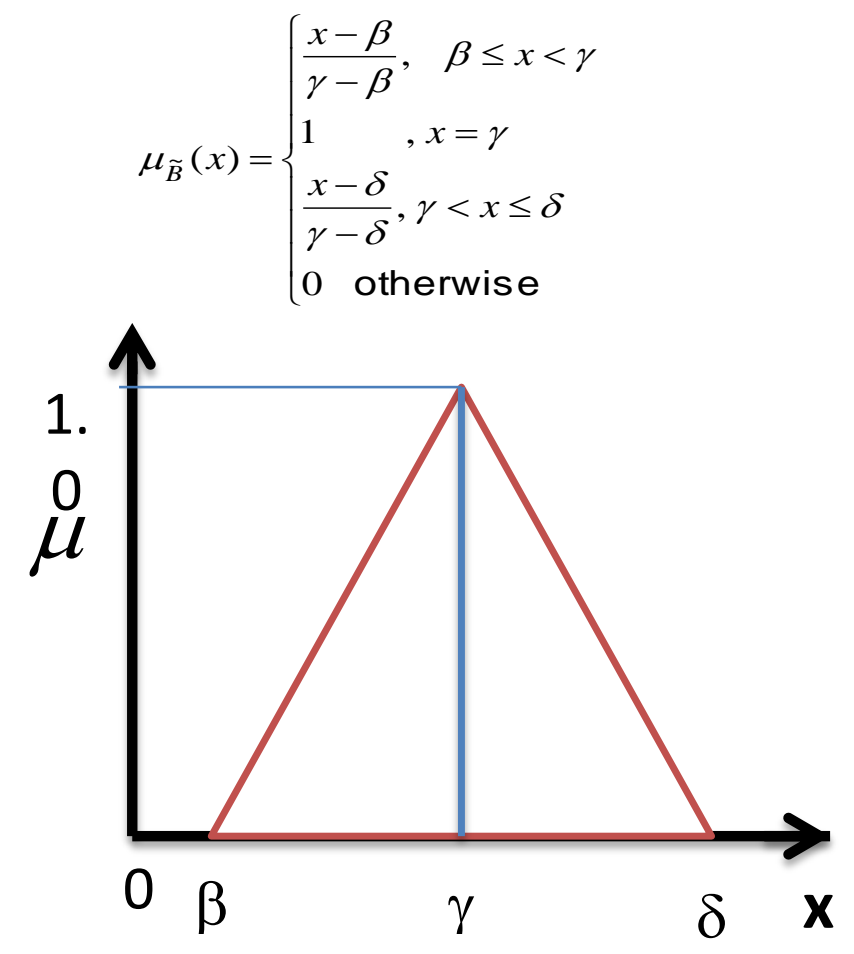

Figure 1. Triangular Fuzzy Number 
Definition 3.1.4 The $\alpha$-cut of a fuzzy number being an interval, the interval arithmetic operation of two fuzzy numbers A and B at its $\alpha$-cut representation is given by

1. Addition : $\left[A_{L}, A_{U}\right]_{\alpha}+\left[B_{L}, B_{U}\right]_{\alpha}=\left[A_{L}+B_{L}, A_{U}+B_{U}\right]_{\alpha}$

2. Subtraction: $\left[A_{L}, A_{U}\right]_{\alpha}-\left[B_{L}, B_{U}\right]_{\alpha}=\left[A_{L}-B_{U}, A_{U}-B_{L}\right]_{\alpha}$

3. Multiplication : $\left[A_{L}, A_{U}\right]_{\alpha} \times\left[B_{L}, B_{U}\right]_{\alpha}=[\min (C), \max (C)]_{\alpha}$, where,

$$
C=A_{L} B_{L}, A_{U} B_{U}, A_{L} B_{U}, A_{U} B_{L}
$$

4. Division $:\left[A_{L}, A_{U}\right]_{\alpha} \div\left[B_{L}, B_{U}\right]_{\alpha}=\left[A_{L}, A_{U}\right]_{\alpha} \times\left[1 / B_{U}, 1 / B_{L}\right]_{\alpha}=$ $[\min (D), \max (D)]_{\alpha}$,

$$
D=A_{L} / B_{U}, A_{U} / B_{U}, A_{L} / B_{L}, A_{U} / B_{L}
$$

\subsection{Formulation of Finite difference scheme}

In this work, the explicit finite difference scheme of the governing Eq. (5) with alpha cut representation of the fuzzy parameters (diffusion coefficient $\tilde{D}$, flow velocity $\tilde{v}$, and all other parameters of $\mathrm{G}$ except $\lambda$ ) has been used. Alpha cut representation of the governing Eq. (5) can be written as

$$
\frac{\partial C_{\alpha}}{\partial t}=D_{\alpha} \cdot \frac{\partial^{2} C_{\alpha}}{\partial x^{2}}-v_{\alpha} \frac{\partial C_{\alpha}}{\partial x}-\lambda C_{\alpha}+G_{\alpha}
$$

We have used the forward time and central space (FTCS) scheme for discretization of the time derivative and space derivative terms $\left(\frac{\partial \tilde{C}}{\partial t}, \frac{\partial^{2} \tilde{C}}{\partial x^{2}}\right.$ and $\left.\frac{\partial \tilde{C}}{\partial x}\right)$ respectively. Since alpha cut representation of the fuzzy parameters are represented as bounded closed interval and positive definite, explicit finite difference scheme used on the alpha cut representation of the radon concentration for its lower and upper bound can be written as

$$
\begin{aligned}
& {\left[C_{i, j+1}^{L}\right]_{\alpha}=\left[\left(\frac{D_{L} k}{h^{2}}+\frac{v_{L} k}{h}\right) C_{i-1, j}^{L}+\left(1-\lambda k-\frac{2 D_{L} k}{h^{2}}\right) C_{i, j}^{L}+\left(\frac{D_{L} k}{h^{2}}-\frac{v_{L} k}{h}\right) C_{i+1, j}^{L}\right]_{\alpha}+G_{\alpha} k} \\
& {\left[C_{i, j+1}^{U}\right]_{\alpha}=\left[\left(\frac{D_{U} k}{h^{2}}+\frac{v_{U} k}{h}\right) C_{i-1, j}^{U}+\left(1-\lambda k-\frac{2 D_{U} k}{h^{2}}\right) C_{i, j}^{U}+\left(\frac{D_{U} k}{h^{2}}-\frac{v_{U} k}{h}\right) C_{i+1, j}^{U}\right]_{\alpha}+G_{\alpha} k}
\end{aligned}
$$

where indices $\mathrm{i}$ and $\mathrm{j}$ refer to the discrete step lengths $\mathrm{h}=\Delta \mathrm{x}$ and $\mathrm{k}=\Delta \mathrm{t}$ for the coordinate $\mathrm{x}$ and time $\mathrm{t}$, respectively. The lower and upper bounds of all the fuzzy parameters at its any $\alpha$-cut representation can be written as $\left[D_{L}, D_{U}\right]_{\alpha},\left[v_{L}, v_{U}\right]_{\alpha},\left[G_{L}, G_{U}\right]_{\alpha}$ respectively. The alpha cut representation of the radon generation rate, $G$ can be written using Eq. (1) as

$$
G_{\alpha}=[f]_{\alpha}\left[\rho_{s}\right]_{\alpha}\left[A_{R a}\right]_{\alpha} \lambda\left(1-[\phi]_{\alpha}\right) /[\phi]_{\alpha}
$$

Therefore, the explicit form of alpha cut value of $\mathrm{G}$ as bounded closed interval can be written as 


$$
\left[G_{L}, G_{U}\right]_{\alpha}=\frac{\left[f_{L}, f_{U}\right]_{\alpha} \times\left[\rho_{s}^{L}, \rho_{s}^{U}\right]_{\alpha} \times\left[A_{R a}^{L}, A_{R a}^{U}\right]_{\alpha} \times\left([1,1]-\left[\phi_{L}, \phi_{U}\right]_{\alpha}\right) \times \lambda}{\left[\phi_{L}, \phi_{U}\right]_{\alpha}}
$$

Therefore, alpha cut value of $\mathrm{G}$ is evaluated by using Eq.(1) and the interval arithmetic (multiplication) operation (see Definition 3.4) of the fuzzy parameters, viz. (f, $\rho, A_{R a}$ and $\phi)$. Equation (8) and (9) represent formulae for lower and upper bounds of the radon concentration at the $(i, j+1)^{\text {th }}$ mesh points for any $\alpha$-cut in terms of known values along the $(i-1)^{\text {th }},(i)^{\text {th }},(i+1)^{\text {th }}$ distances and at $j^{\text {th }}$ time. In this way, the truncation error for the difference Eq. (8) and Eq. (9) are reduced to $\mathrm{O}\left(\mathrm{k}, \mathrm{h}^{2}\right)$. But, if one uses forward difference scheme for all derivatives in Eq. (5), the truncation error is larger and its value is $\mathrm{O}(\mathrm{k}, \mathrm{h})$. Using a small enough value of $\mathrm{k}$ and $\mathrm{h}$, the truncation error can be reduced until the accuracy achieved is within the error tolerance [16]. The finite difference forms of the initial condition for lower and upper bound of the radon concentration are given by

$$
C_{i, o}^{L}=0 \text { and } \quad C_{i, o}^{U}=0 \quad \text { for } \mathrm{t}=0 ; 0<\mathrm{x}<\mathrm{L}
$$

The finite difference forms of the boundary conditions for lower and upper bound of the radon concentration are given by

$$
\begin{array}{cc}
C_{0, j}^{L}=C_{0, j}^{U}=C_{0} & \text { for } \mathrm{x}=0 ; \mathrm{t}>0, \\
C_{N, j}^{L}=C_{N, j}^{U}=0 & \text { for } \mathrm{x}=\mathrm{L} ; \mathrm{t}>0
\end{array}
$$

where $\mathrm{N}=\mathrm{L} / \mathrm{h}$ is the grid dimension in the $\mathrm{x}$ direction. $\mathrm{L}$ is the total length of the soil slab considered for the computation.

\section{Uncertainty Modeling and Algorithm of FUZYRAD}

Uncertainty of concentration of radon is mainly due to the imprecision in measurements of the radon diffusion coefficient and flow velocity of radon in air. As fuzzy set theory is the most appropriate tool for quantifying uncertainty associated with imprecision in measurement, imprecise value of the parameters of interest (diffusion coefficient and flow velocity of radon in air) are represented in terms of a fuzzy number. The uncertainty due to imprecision (insufficient measurements) or lack of information is known as epistemic uncertainty $[17,18]$. Hence in this work uncertainty modeling of concentration of radon describing its transport from subsurface into buildings is based on fuzzy set theoretic approach. Accordingly, radon concentration results as a fuzzy number [19] and hence the $\alpha$-cut representation of the radon concentration has been computed using explicit method of finite difference in presence of the fuzziness of the radon diffusion coefficient and flow velocity of radon in air. The membership function of the radon concentration results as a generalized triangular fuzzy number. By applying uncertain (fuzzy) parameters such as radon diffusion coefficient $D$ and mean air velocity $v$ in the analytical steady state solution of the same problem (without the term G) given by Telford (1983) [20], uncertainty of the radon concentration as a triangular fuzzy number can be written as

$$
\tilde{C}=C_{0} \exp \left[\frac{(L-x) \tilde{v}}{2 \tilde{D}}\right] \times \frac{\sinh \left(x \sqrt{\frac{\tilde{v}^{2}}{4 \tilde{D}^{2}}+\frac{\lambda}{\tilde{D}}}\right)}{\sinh \left(L \sqrt{\frac{\tilde{v}^{2}}{4 \tilde{D}^{2}}+\frac{\lambda}{\tilde{D}}}\right)}
$$


In this work, the uncertainty of the radon concentration using the Eq. (14) is used to test the author's fuzzy finite difference numerical scheme based uncertainty results of the radon concentration. Uncertainty output in both the cases is expressed in terms of the $\alpha$ cut representation as lower and upper bound for any specified $\alpha$-cut. The degree of uncertainty [21] of radon concentration is given by

$$
\left.\Delta C\right|_{\alpha}=\left[\frac{C^{U}-C^{L}}{C^{U}+C^{L}}\right]_{\alpha}
$$

where, $C^{L}$ and $C^{U}$ are the alpha cut representation of lower and upper bound of the radon concentration. Section 4.1 presents an algorithm used for computing the alpha cut value of the radon concentration.

\subsection{Algorithm of FUZYRAD}

Algorithm developed for the present work is based on fuzzy finite difference (FTCS) scheme with fuzzy parameters as the coefficient of the governing partial differential equation. This algorithm is implemented in our developed code 'FUZYRAD (version 1.0)' Partial differential equation used here is known as advection diffusion equation which has been written in one dimension form (Eq. (5)). Fuzziness of the fuzzy parameters is expressed by a triangular membership function (see Eq. (7)). Alpha cut value of fuzzy parameters for each alpha ranged from 0 to 1 with an increment of 0.1 is represented as an interval as [Lower bound, Upper bound]. Finite differencing of radon transport equation is generated for each lower and upper bound corresponding to each alpha. Numerical solution of each such difference equation at any space-time mesh point is evaluated independently. On the basis of this outline, our algorithm is as follows:

Step 1: Notify the measurement uncertainty of the parameters as (mean $\pm \sigma$ )

Step 2: Fuzzify the uncertain parameters as triangular fuzzy number by Eq. (7) as

$$
(\beta=\text { mean }-\sigma, \gamma=\text { mean, } \delta=\text { mean }+\sigma)
$$

Step 3: Construct the alpha cut value of each fuzzy parameters:

$$
D_{\alpha \in[0,1]}=\left[D_{L}, D_{U}\right]_{\alpha} \quad \text { and } \quad v_{\alpha \in[0,1]}=\left[v_{L}, v_{U}\right]_{\alpha} ;
$$

Step 4: Compute $\mathrm{h}=\Delta \mathrm{x}=\mathrm{L} / \mathrm{N}$ and $\mathrm{k}=\Delta \mathrm{t}=\mathrm{T}$ (total time of simulation)/M (Total number of time grid points)

Step 5: Compute G using Eq. (1)

Step 6: Compute the lower and upper bound of radon concentration for each $\alpha \in[0,1]$ using corresponding lower and upper bound of parameters $\tilde{D}$ and $\tilde{v}$ (see Eq. (8) and Eq. (9)).

Step 7: Compute the degree of uncertainty using Eq. (15).

\section{Results and Discussion}

In order to obtain the uncertainty of the radon concentration due to the fuzziness of the radon diffusion coefficient and flow velocity of radon in air, it has been assumed that one side of the soil slab $(\mathrm{x}=\mathrm{L})$ is exposed to constant ${ }^{222} \mathrm{Rn}$ concentration of $C_{0}=10^{5} \mathrm{~Bq} / \mathrm{m}^{3}$. The domain of computation is considered as a soil slab geometry of $10 \mathrm{~m}$ deep in the vertical direction and this is shown in Figure 2. 


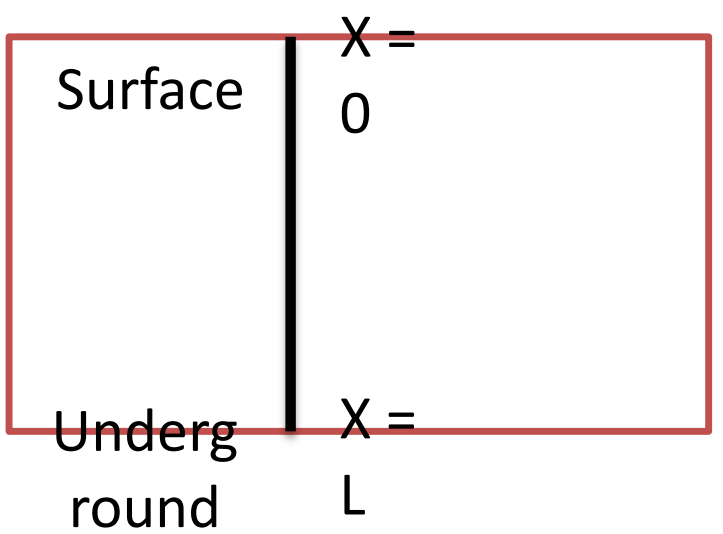

\section{Figure 2. Radon Transport Through Soil Slab from the underground $(x=L)$ to surface $(x=0)$}

The fuzziness of the model parameters are expressed as triangular fuzzy number because the imprecision result is around the most likely value (experimental measured mean). Accordingly, the triangular fuzzy number of the radon diffusion coefficient, $\tilde{D}$ $\left(\mathrm{m}^{2} / \mathrm{s}\right)=\langle 2.4 \mathrm{E}-06,4.0 \mathrm{E}-06,5.6 \mathrm{E}-06\rangle$ (most likely value of the diffusion coefficient is the measured value for clay soil with a few samples and lower and upper extremes are due to its imprecision in measurement), velocity of radon in air, $\tilde{v}(\mathrm{~m} / \mathrm{s})=<8.0 \mathrm{E}-7,1.0 \mathrm{E}-6$, 1.2E-6> (most likely value of the velocity is typical radon-bearing advective air velocity in clay soil due to thermo gradient in the slab), radon emanation coefficient, $f=<0.091$, $0.130,0.169\rangle$, density of the soil, $\rho=\langle 1.855,2.650,3.445\rangle$, activity of radon in soil, $A_{R a}$ $=\langle 42.36,70.60,98.84\rangle$ and porosity of the soil, $\epsilon=\langle 0.20,0.25,0.30\rangle$. The membership function of the model parameters such as diffusion coefficient and velocity are only shown in Figures 3 and 4. Uncertainty of the radon concentration is expressed in terms of a closed interval bounded by lower and upper value of radon concentration. In order to have a stability in the numerical calculation of each lower and upper bound of radon concentration corresponding to lower and upper bound of model fuzzy parameters for any $\alpha$-cut, step lengths, $\Delta \mathrm{x}=\mathrm{h}=0.2 \mathrm{~m}$ and $\Delta \mathrm{t}=\mathrm{k}=10 \mathrm{~s}$ are taken into consideration. Both the process of diffusion and advection are considered in the calculation. The uncertainty of the radon generation rate has been computed separately using its alpha cut representation as given by Eq. (10). The membership function of the radon generation rate, $\mathrm{G}$ is shown in Figure 5. In order to test the stability of the finite difference scheme with the step lengths $\Delta \mathrm{x}$ and $\Delta \mathrm{t}$ as mentioned, numerical calculation of radon concentration profile with the most likely value of the model parameters (deterministic or crisp) has been carried out and the results are compared with corresponding analytical result. Figure 6 shows the comparison of both numerical and analytical profile of radon concentration. 


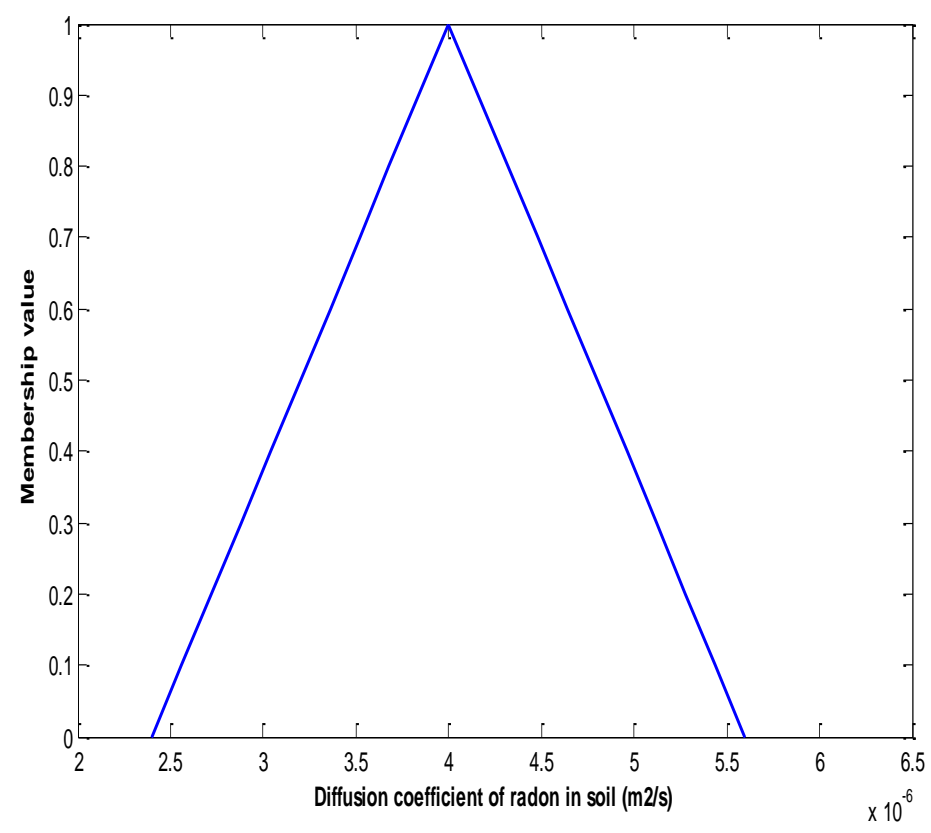

Figure 3. Triangular Membership Function of Diffusion Coefficient Affiliation

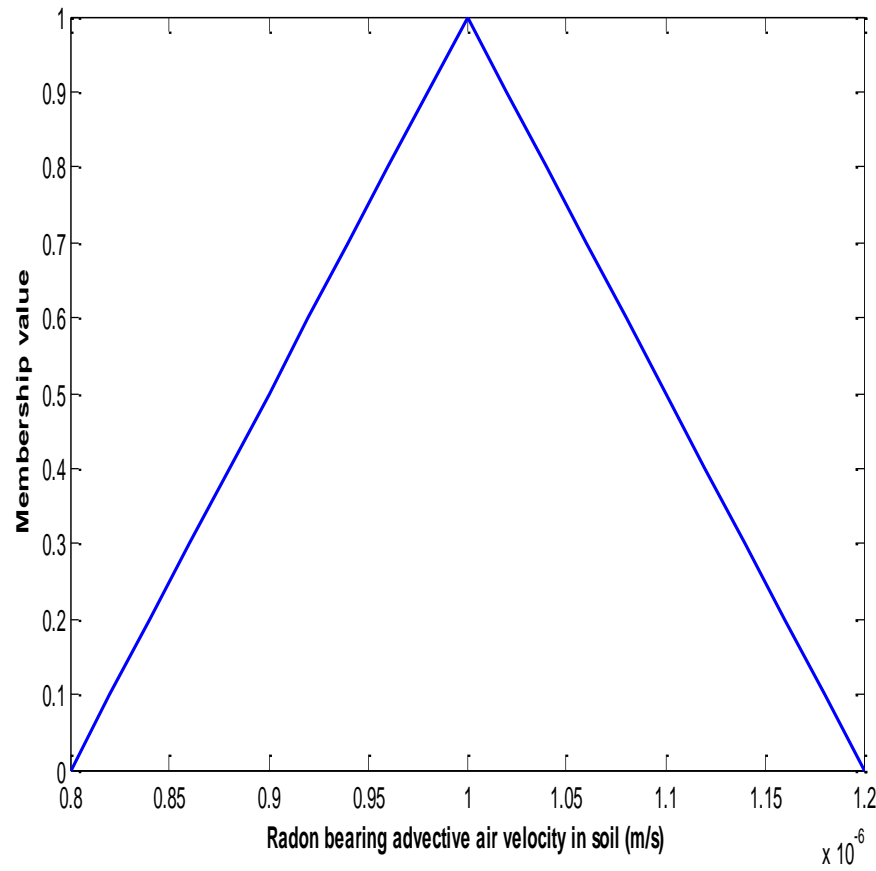

Figure 4. Triangular Membership Function of Flow Velocity 


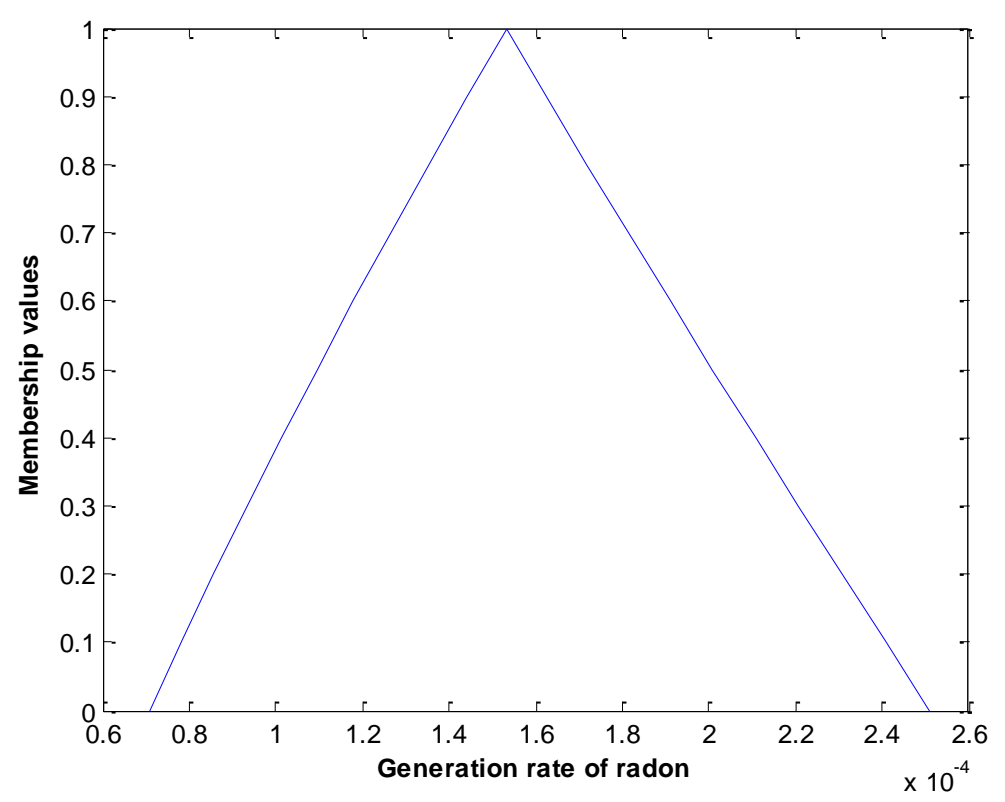

Figure 5. Membership Function of Generation Rate of Radon

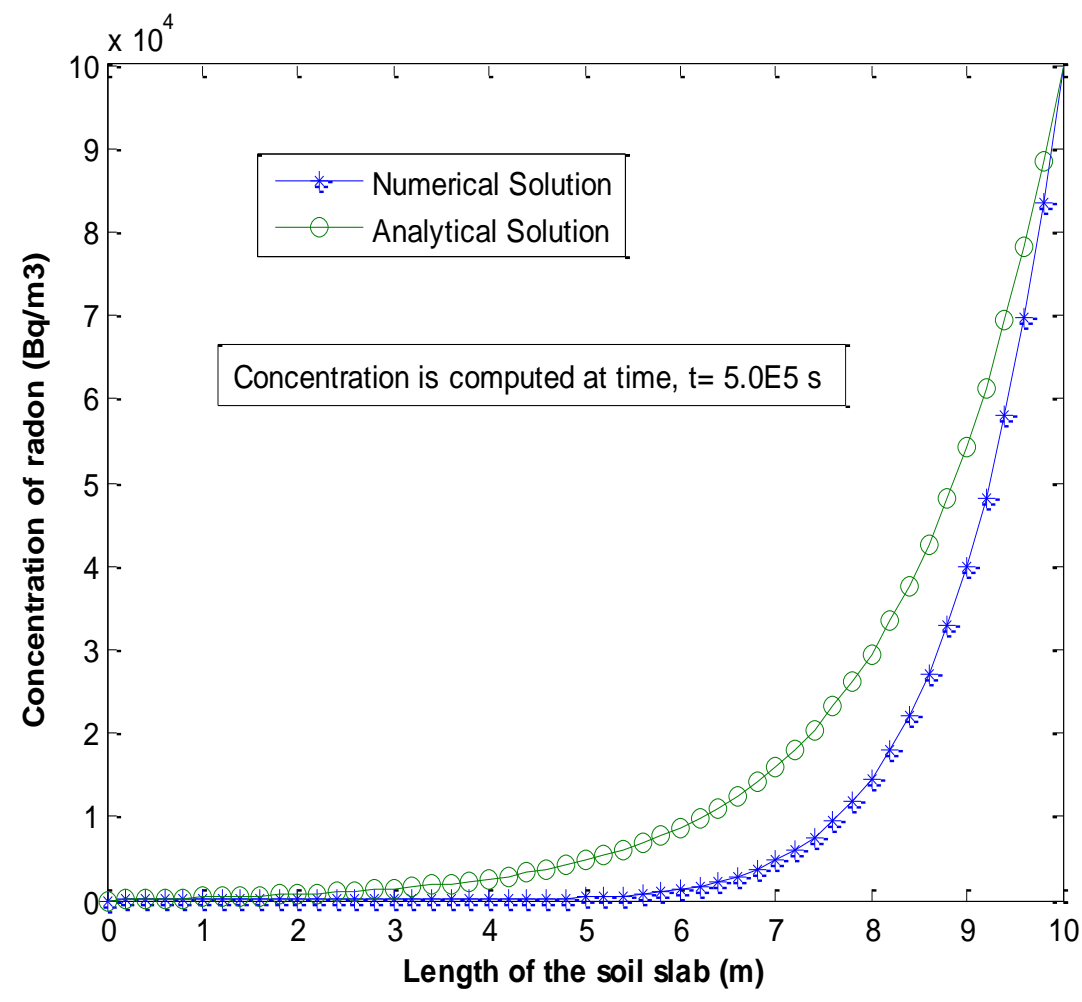

Figure 6. Concentration (Numerical and Analytical) Profile of Radon in Soil Slab with Deterministic Value of Parameters (Diffusion Coefficient and Flow Velocity)

The numerical calculation of the lower and upper bound of the radon concentration for a specific time and at each length of the soil slab (say at the end of the total time of simulation) results the fuzziness of the radon concentration. The fuzziness of the radon concentration at lengths, $x=7.0 \mathrm{~m}, 7.8 \mathrm{~m}, 9.0 \mathrm{~m}$ and $9.8 \mathrm{~m}$ for a specific time $(\mathrm{t}=$ 
5.0E5 seconds) is represented in terms of the membership function of the radon concentration and the results are shown in Figures 7a-7d.

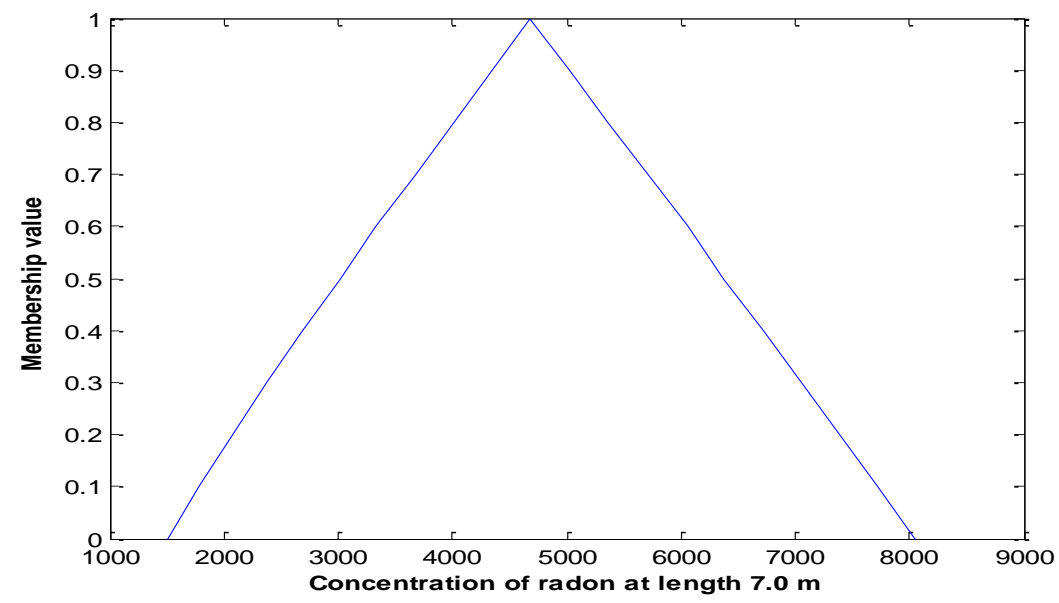

Figure 7a. Membership Function of Radon Concentration at Length $7.0 \mathrm{~m}$

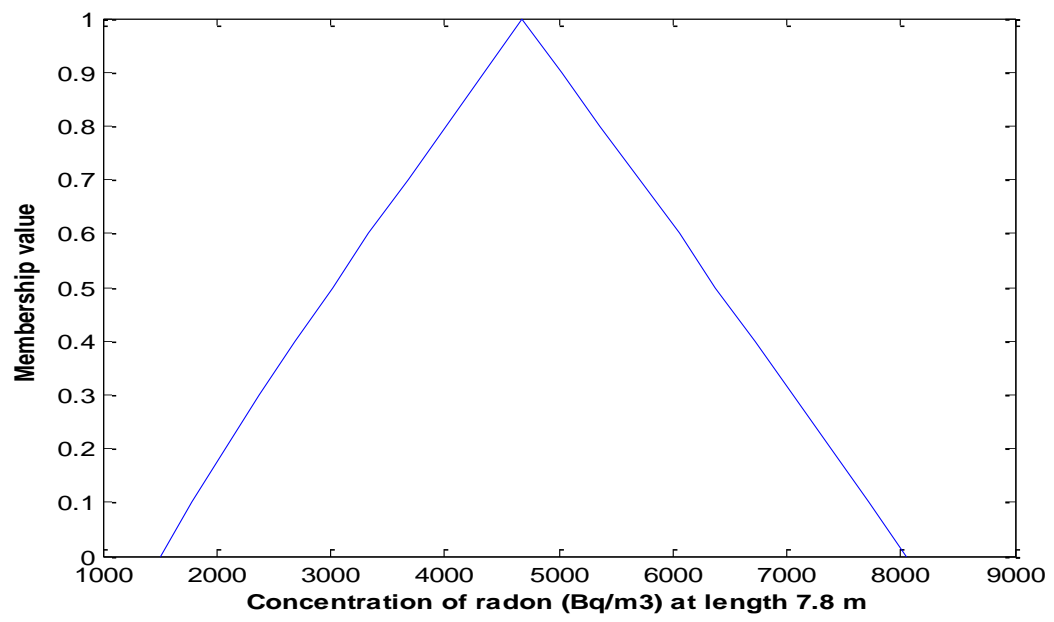

Figure 7b. Membership Function of Radon Concentration at Length $7.8 \mathrm{~m}$

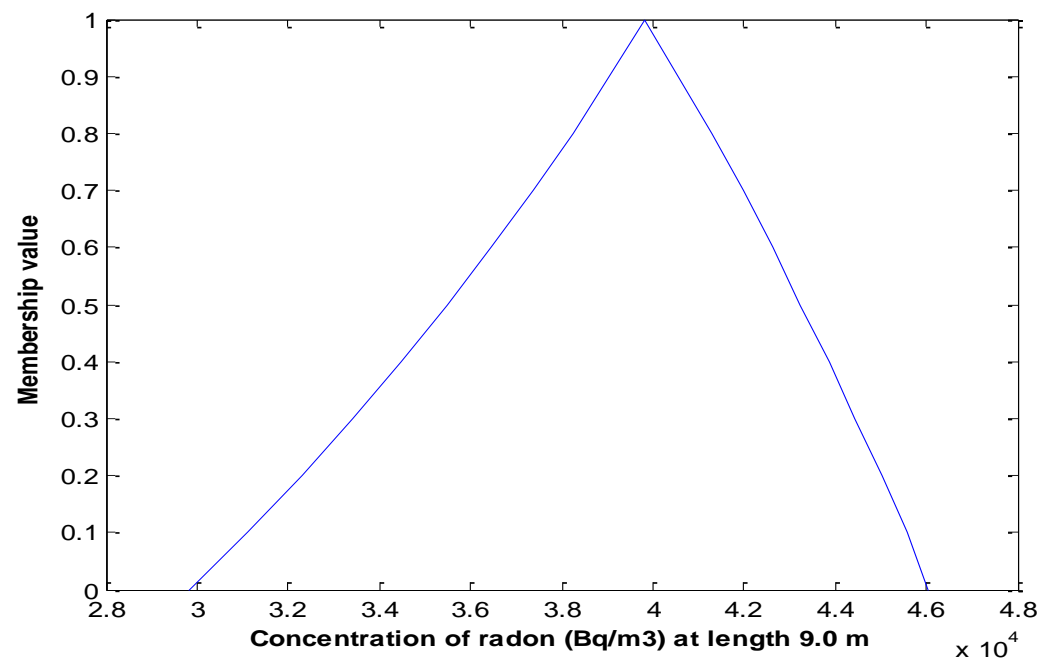


Figure 7c. Membership Function of Radon Concentration at Length $9.0 \mathrm{~m}$

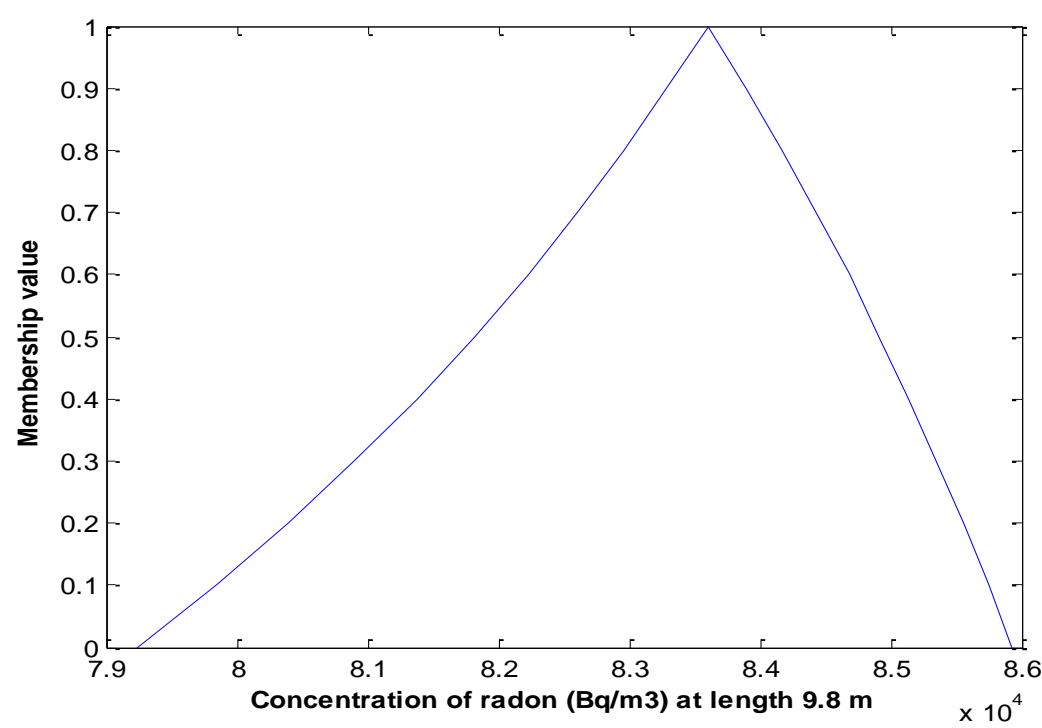

Figure 7d. Membership Function of Radon Concentration at Length $9.8 \mathrm{~m}$

Finally, the lower bound, most likely value and upper bound of radon concentration for various lengths $(\mathrm{x}=0,1,2, . .10 \mathrm{~m})$ of the domain of computation at any alpha cut of 0.5 for the total time of simulation (5.0E5 s) is computed and the corresponding result is presented in Figure 8. It can be observed from Figure 8 that the transport of radon from subsurface into building results an uncertainty (due to fuzziness of the model parameters) in radon concentration mostly at lengths ranging from $7 \mathrm{~m}$ to $9 \mathrm{~m}$ within the range of the computational domain as $10 \mathrm{~m}$. In order to defend author's fuzzy finite difference numerical scheme, lower and upper bound of radon concentration profile using Eq. (14) at alpha cut $=0.5$ and 0.8 are computed and shown in Figures 9 and 10. Membership function of the radon concentration using Eq. (14) at length of the slab, $\mathrm{x}=7.8 \mathrm{~m}$ and $\mathrm{x}=$ $9.8 \mathrm{~m}$ are also computed and the corresponding results are plotted in Figs. 11 and 12 respectively. The degree of uncertainty at length $8 \mathrm{~m}$ for an alpha cut of 0.5 is estimated using Eq. (13) as 0.242.

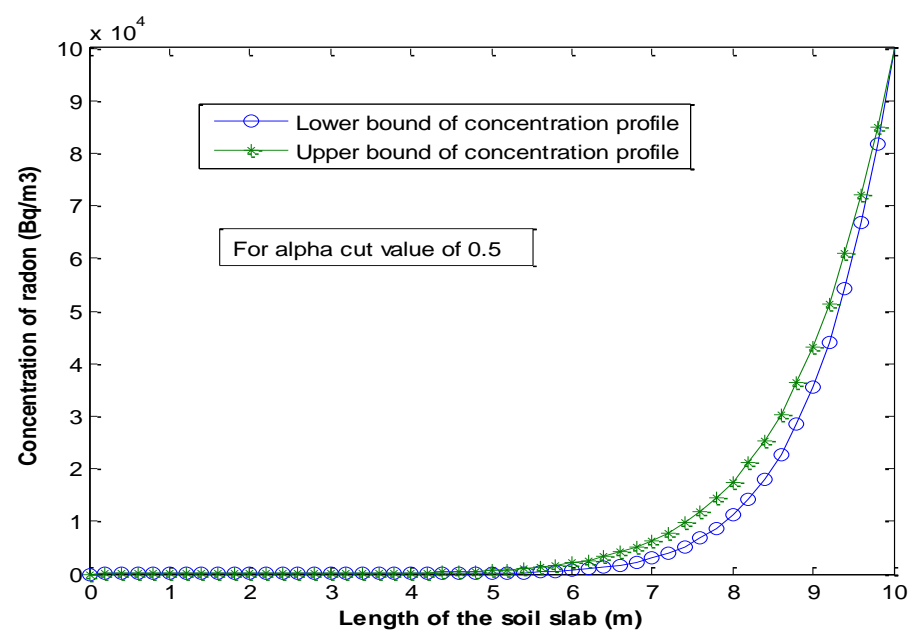

Figure 8. Profile of Uncertainty (Lower and Upper Bound) in Radon Concentration at Alpha Cut of 0.5 


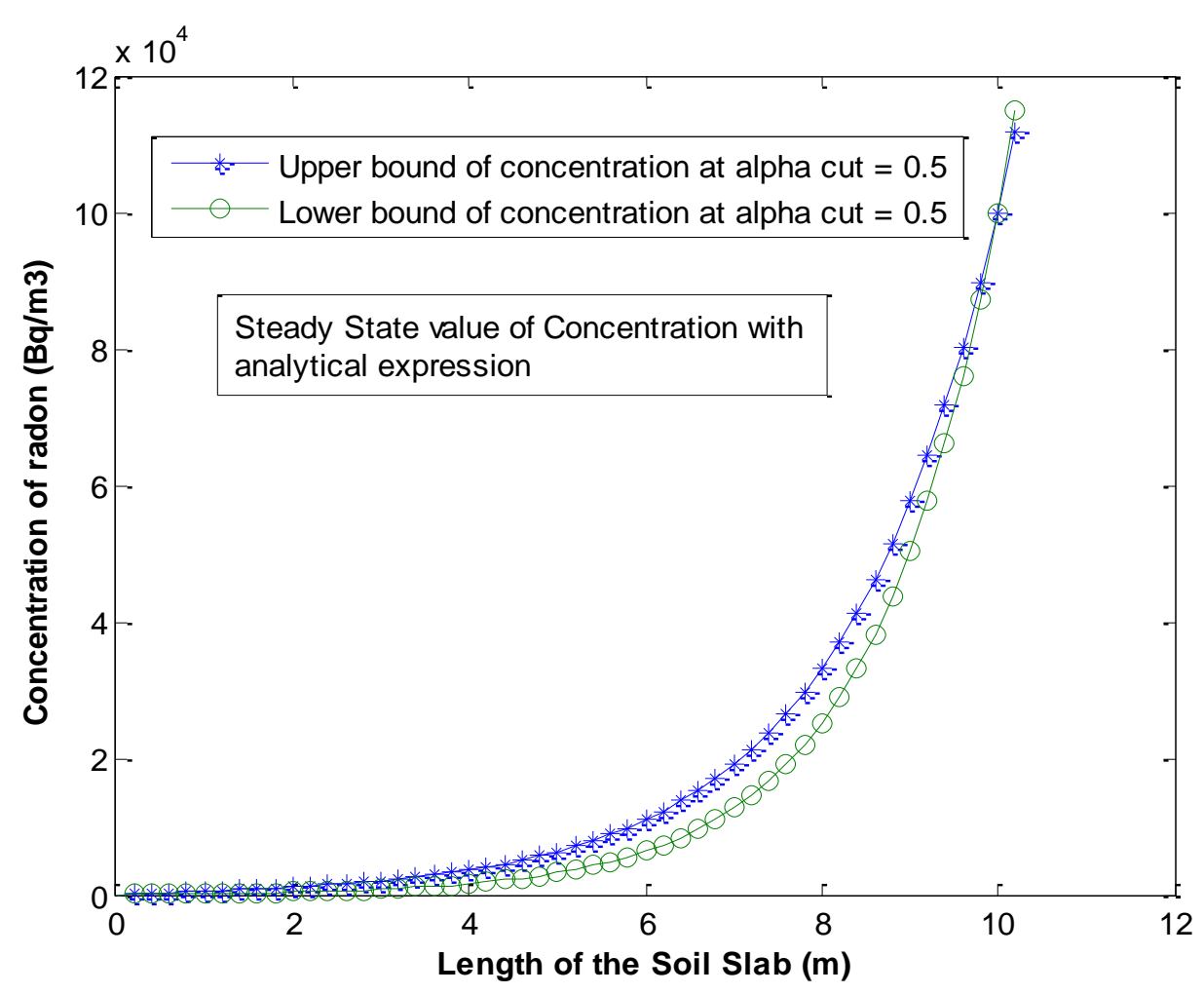

Figure 9. Profile of Uncertainty (Lower and Upper Bound) in Radon Concentration at Alpha Cut of 0.5 with Analytical Expression [Eq. (14)]

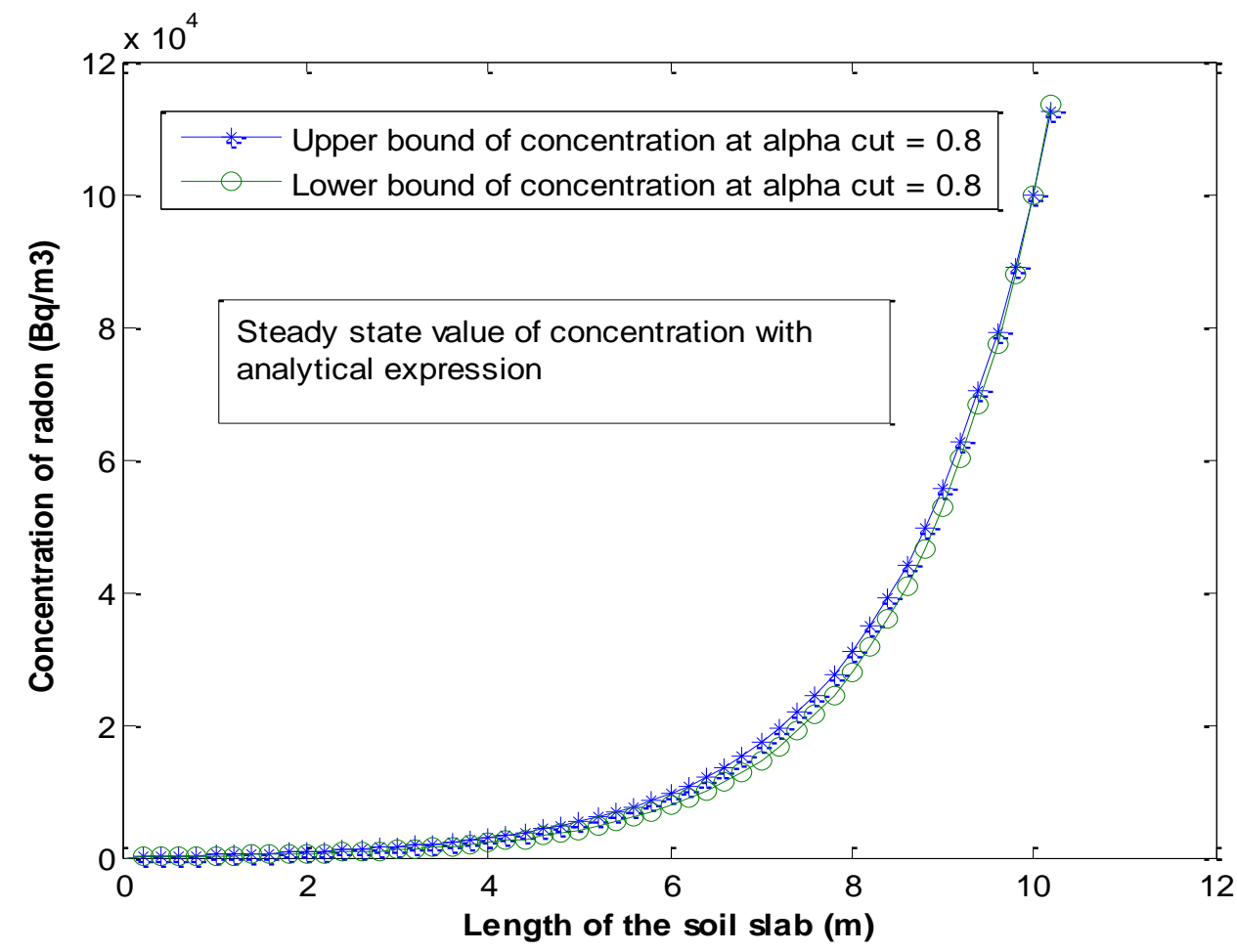

Figure 10. Profile of Uncertainty (Lower and Upper Bound) in Radon Concentration at Alpha Cut of 0.8 with Analytical Expression [Eq. (14)] 


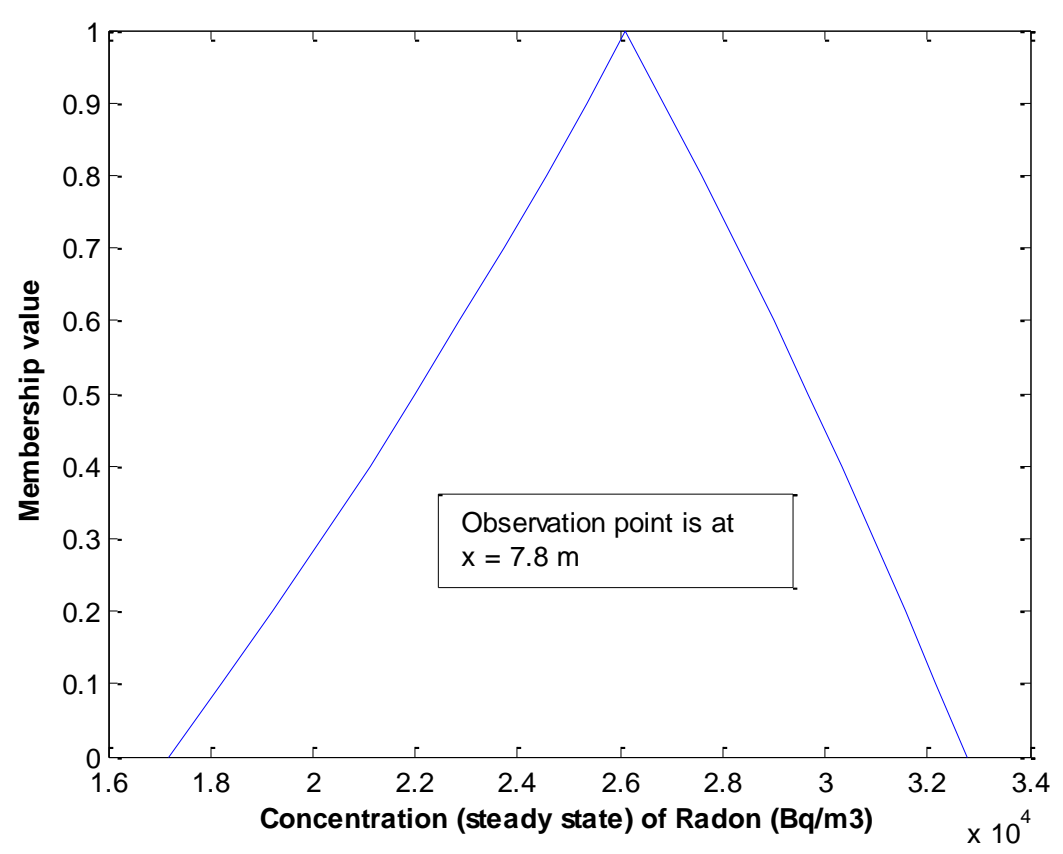

Figure 11. Membership Function of Steady State Radon Concentration using Eq. (14) at Length $7.8 \mathrm{~m}$

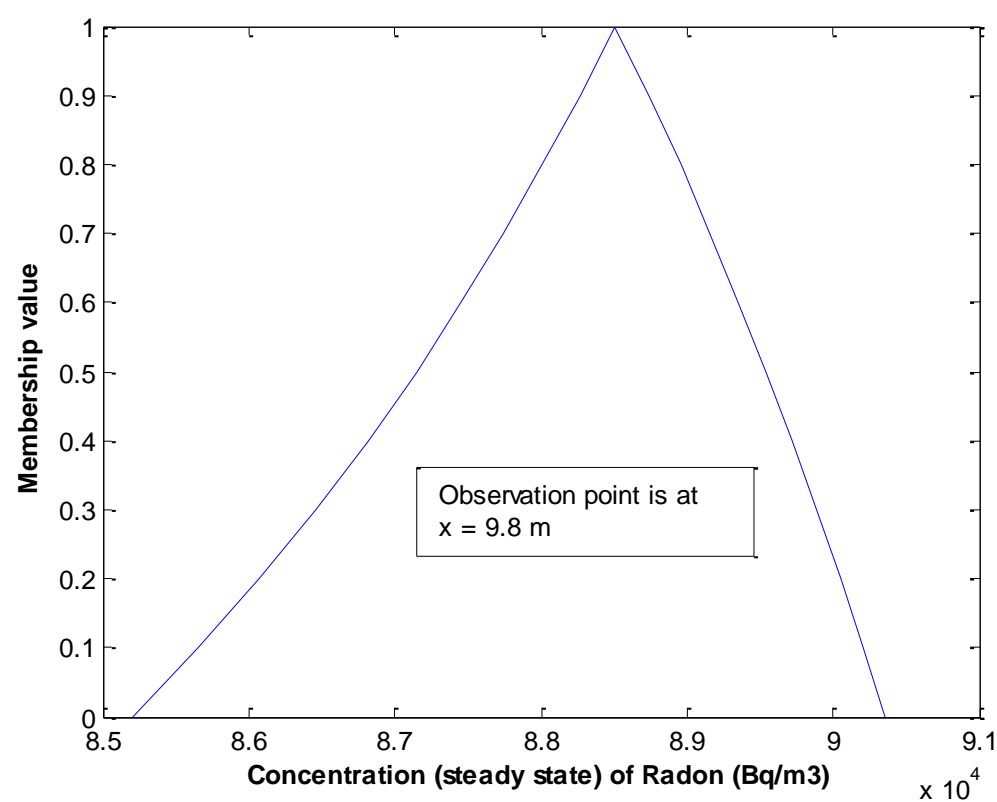

Figure 12. Membership Function of Steady State Radon Concentration using Eq. (14) at Length $9.8 \mathrm{~m}$

\section{Conclusions}

Fuzzy finite difference method based numerical solution of the radon transport equation in presence of a fuzziness in its coefficient parameters (diffusion coefficient and flow velocity in soil) is presented for investigating the process of ${ }^{222} \mathrm{Rn}$ transport through subsurface soil modeled in the form of a rectangular slab. Measurement uncertainty of diffusion coefficient and flow velocity of radon in soil leads to them as fuzzy and their 
fuzziness are described by triangular fuzzy number because, most likely value (measured mean) of these parameters are circumvented by the corresponding boundaries (left and right bounds) as mean \pm error. Membership function of these fuzzy numbers has been utilized to generate the respective alpha cut value of these fuzzy numbers. Alpha cut value of a fuzzy number being an interval, the lower and upper bounds are independently adopted for obtaining the numerical solution of one dimension radon transport equation. Basis of the numerical scheme is reported as the explicit finite difference (FTCS) but lower and upper bounds of the coefficient parameters (evolutes from fuzziness through alpha cuts) of the parent equation justifies the name fuzzy finite difference. Uncertainty of the radon concentration at any spatial location for a fixed time (say, total time allowed for simulation) is reported in terms of the alpha cut value of the radon concentration.

It has been shown that FFDM is effective and accurate for not only solving the radon transport equation with fuzziness in diffusion coefficient and in flow velocity but also provides a tool for estimating the uncertainty associated with the concentration of radon. The uncertainty of radon concentration as estimated is especially important to obtain the uncertainty in the inhalation exposure by propagation through the uncertainty of the breathing rate and dose conversion coefficient.

\section{References}

[1] T. Ren, "Source, level and control of indoor radon", Radiat. Prot., vol. 21, no. 5, (2001), pp. 291-297.

[2] J. C. H. Miles and J. D. Appleton, "Mapping variation in radon potential both between and within geological units", J. Radiol. Prot., vol. 25, no. 257, (2005).

[3] V. C. Rogers and K. K. Nielson, "Multiphase radon generation and transport in porous materials", Health Physics, vol. 60, no. 807, (1991).

[4] G. lelsch, C. Ferry, G. Tymen and M. C. Robe, "Study of a predictive methodology for quantification and mapping of the radon-222 exhalation rate", Journal of Environmental Radioactivity, vol. 63, no. 15, (2002).

[5] V. G. Escobar, F. V. Tome and J. C. Lozano, "Proceedings for the determination of ${ }^{222}$ Rn exhalation and effective ${ }^{226} \mathrm{Ra}$ activity in soil samples”, Appl. Radiat. Isot., vol. 50, no. 6, (1999), pp. 1039-1047.

[6] V. Moreno, C. Baixeras, L. Font and J. Bach, "Indoor radon levels and their dynamics in relation with the geological characteristics of La Garrotxa, Spain”, Radiation Measurements, vol. 43, no. 1532, (2008).

[7] B. L. Cohen, "Methods for predicting the effectiveness of uranium mill tailings covers", Nucl. Instrum. Methods, vol. 164, (1979), pp. 595-599.

[8] T. Sasaki, Y. Gunji and T. Lida, "Transient-diffusion measurements of radon: practical interpretation of measured data", Nucl. Sci. Technol., vol. 44, no. 7, (2007), pp. 1032-1037.

[9] J. Haijing, G. E. Liangquan, L. Yanchang and G. Yi, "Preliminary study on a reginal radon concentration in surface soil prediction method", Progress in Nuclear Science and technology, vol. 1, (2011), pp. 364-367.

[10] Medici, F. and Rybach, L., Measurements of indoor radon concentrations and assessment of radiation exposure., J. Appl. Geophys., 31:153-163, 1994.

[11] Telford, W.M., Radon mapping in the search for uranium. In: A.A. Fitch (Editor), Developments in Geophysical Exploration Methods, vol. 4, Elsevier Barking, pp. 155-194, 1983.

[12] Uro sevic, V., Nikezic, D., Radon transport through concrete and determination of its diffusion coefficient. Radiat. Prot. Dosim. 104 (1), 65-70, 2003

[13] Savovic', S., Djordjevich, A., Numerical solution of the diffusion equation describing the flow of radon through concrete. Appl. Radiat. Isot. 66 (4), 552-555, 2008.

[14] Nazaroff, W.W. and Sextro, R.G., Technique for measuring the indoor ${ }^{222} \mathrm{Rn}$ source potential of soil. Environ. Sci. Technol., 23(4):451, 1989.

[15] Zadeh L.A., Fuzzy Sets, Information and Control, 8:338-353, 1965.

[16] Anderson, J. D., Computational Fluid Dynamics. McGraw-Hill, 1995.

[17] Bilal M. Ayyub and George J Klir, Uncertainty Modelling and Analysis In Engineering and The Sciences, Chapman and Hall/CRC Press, Boca Ratan, 2006.

[18] Rituparna Chutia, Supahi Mahanta and D. Datta,, Sensitivity analysis of atmospheric dispersion model RIMPUFF using the Hartley-like measure, J. Appl. Math. \& Informatics, vol. 31, no. 1 - 2, pp. 99 - 110, 2013.

[19] Klir, G. J. and Yuan, B., Fuzzy sets and Fuzzy Logic: Theory and Applications, Prentice Hall, PTR, Upper Saddle River, NJ, 1995.

[20] Telford, W. M., Radon mapping in the search for uranium, In: Developments in Geophysical Exploration Methods, vol. 4, Fitch, A. A., Ed., pp. 155-194., Elsevier,1983. 
International Journal of Energy, Information and Communications

Vol.6, Issue 4 (2015)

[21] McKone T.E., and Bogen K. T., Predicting the uncertainties in risk assessment. Environ. Sci. Technol. 25(10): 674-1681, 1991. 